\title{
Multi-scale Texture in SAR Imagery: Landscape Dynamics of the Pantanal, Brazil
}

\author{
Hermann J. H. Kux and Geoffrey M. Henebry" \\ Instituto Nacional de Pesquisas Espaciais \\ Caixa Postal 515 \\ 12227-010 São José dos Campos SP Brazil \\ T: 55.123.418977 F: 55.123.218743 \\ hermann@ltid.inpe.br \\ "Kansas State University \\ Division of Biology/Ackert Hall \\ Manhattan KS 66506 USA \\ T: 913.532.7997 F:913.532.6653 \\ gmh@ andro.konza.ksu.edu
}

Abstract

The potential for environmental monitoring of natural landscapes using radar remote sensing is great. However, to realize this potential, new tools for radar image analysis are needed. Landscapes that exhibit complex spatiotemporal variability in terms of backscattering cannot be well characterized using standard approaches to texture. We assess three methods for multi-scale texture measurement: lacunarity, diversity, and GLCM measures. We used three ERS-1 SAR images of the Nhecolândia region of the Brazilian Pantanal from December 1992 to May 1993. Lacunarity and diversity measures captured the seasonal transformation of the landscape due to rising floodwaters; GLCM measures did not, however, exhibit much sensitivity to either anisotropies or temporal differences in images. In addition, lacunarity analysis was able to distinguish between speckle-generated texture from texture produced by scene object backscattering.

\section{INTRODUCTION}

Environmental monitoring of vegetated landscapes will increase during the decade as data from spacebome SAR platforms (ERS-2, J-ERS, RADARSAT) become available. Scene models (sensu Strahler et al. 1986) for radar remote sensing have focussed primarily ón deterministic, bottomup descriptions of backscattering from static scene objects. The dynamic nature of environmental monitoring, however, necessitates the development of spatio-temporal scene models that describe image phenomenology (empirical, top-

down) and permit linkages to other dataforms, ө.g., GIS, simulation models, optical imagery (Henebry 1993).

Texture analysis provides a first step toward developing dynamic scene models for SAR imagery. Several studies have investigated the efficacy of texture measures in SAR image segmentation and classification using either autocorrelation/autocovariance formulae derived from simple phenomenological models of backscattering (e.g., Sheen and Johnston 1992; Rignot and Kwok 1993) or measures based on the Grey Level Co-occurrence Matrix (GLCM) (e.g., Ulaby et al. 1986; Barber and LeDrew 1991). The GLCM describes probabilities of the cooccurrence of two speclfic grey-levels given specific pixel locations in terms of relative direction and distance (for an overview, see Haralick and Shapiro 1992). Both approaches describe texture in terms of (dis)similarity based on spatial proximity (lag-neighbortood) but they do not include an explicit consideration of scaling effects on spatial pattem and they are predicated on an assumption of spatial stationarity, which is seldom met in natural landscapes. Further, GLCM measures do not explicitly address speckle noise and thus can yield biased estimates of scene texture.

We compared multi-scale GLCM measures against two window-based approaches to texture, diversity and lacunarity. Diversity describes tonal evenness in the image histogram. Although an aspatial measure, it can used to identify critical scales when observed at multiple scales and thus is an implicit measure of scene texture. Lacunarity is a readily calculable metric of spatial nonstationarity. Lacunarity analysis has the power to distinguish between speckle-dominated texture and the texture generated by scene objects. Using ERS-1 SAR imagery, we examined the efficacy of these three techniques in capturing the spatio-temporal dynamics of the Brczilian Pantanal.

\section{STUDY AREA}

The Pantanal is the largest wetland habitat on the planet: an immense alluvial plain formed during the Holocene, it covers $139,000 \mathrm{~km}^{2}$ in Brazilian states of Mato Grosso and Mato Grosso do Sul (Rizzini et al. 1988). The Pantanal may also be the most radiometrically dynamic landscape on the planet due to extensive seasonal flooding by the Rio Paraguai and its tributaries. An ecotonal landscape, the Pantanal is a complex mosaic of shallow lakes, periodically inundated grasslands, and islands and elevated corridors of forest, which together support an abundant and diverse fauna of birds, fish, reptiles, and mammals, including four million head of cattle (Alho et al. 1988).

We used INPE-processed ERS-1 SAR images from the Nhecolandia region of the Pantanal $\left(\mathrm{S} 19.2^{\circ}, \mathrm{W} 56^{\circ}\right)$. Images cover a common area of $590 \mathrm{~km}^{2}$. This area comprises the southern tier of the vast alluvial fan associated with the Rio Taquari and is characterized by hundreds of shallow freshwater and brackish baías. The December image represents the driest portion of the region's annual flooding cycle: baías and islands, beaches and corridors are distinct. By February flooding had commenced but was not yet generalized: previously sharp edges are blurred. The May image represents the flooding peak: widespread growth of aquatic macrophytes and perennial grasses that emerged from areas of freshwater inundation shifts backscattering patterns and homogenize most of the image, but the brackish ba/as remain distinct. 


\section{Lacunarity \\ MULTI-SCALE MEASURES FOR TEXTURE \\ Lacunarity describes the complex intermingling of the} shape and distribution of gaps within an image: a highly lacunar image exhibits gaps distributed across a broad range of sizes (Mandelbrot, 1983; Plotnick et al. 1993). Lacunarity is an aspect of fractal geometry: lacunarity $(L)$ is the multiplicative prefactor in the general power-law formula of which the fractal dimension is the exponent, $F(x)=L x^{(D-E)}$. As a texture measure, lacunarity quantifies the deviation of a geometric object (e.g., shape, pattern, fractal) from translational invariance and thus is well-suited to analysis of natural scenes.

Lacunarity can be estimated by a simple index, $\Lambda$, which is sensitive to observation scale, as is expected from a fractal measure. Indeed, the index's descriptive power is revealed only when it is observed as a function of window size $w$. The maximum value of $\Lambda(w)$ occurs when the window size equals the spatial resolution of the image; it then equals the inverse of the proportion of occupied pixels. Conversely, the minimum value $(=1)$ occurs when the observation window equals the image size, i.e., when the variance is zero. The reader is referred to Plotnick et al. (1993) for background on the calculation of the index. We extend their method here by random resampling the image stratified across window sizes, rather than exhaustive sampling.

Plotting the log of the lacunarity index as a function of the log of window size, the resulting curve illustrates the scale-dependency of spatial nonstationarity. The decay pattern of the lacunarity function contains significant information about the spatial structure of the image (Plotnick et al. 1993). A spatially random image exhibits a rapid decay to the minimum value. An image with selfsimilarity across some range of scales exhibits a linear decay, the slope of which is an estimate of the fractal dimension of the self-similar pattern within that range. For an image with an articulation of objects at a particular scale, the lacunarity decay is slow until the window size exceeds the scale of the objects and is rapid thereafter.

Although lacunarity is defined only for binary data, greyscale data can be analyzed by deriving a series of binary images based on quantiles of the image histogram. Here we construct four binary images from each SAR image corresponding to the quartiles of the histogram. For example, in the first quartile image (Q1) pixels having grey levels between the minimum and the 25th percentile are mapped to white and the remaining pixels are mapped to black. The Q1 image thus illustrates the spatial distribution of the regions in the scene that have the least backscatter (open water); similarly, the Q4 image captures the arrangement of the pixels with the greatest backscatter (trees). Changes in the spatial arrangement of backscattering objects translates into changes in the lacunarity function; thus, lacunarity analysis is useful for characterization of natural spatio-temporal variability within

a scene as well as change detection.

Lacunarity analysis can discriminate between the texture of speckle noise and the texture intrinsic to the scene. Speckle noise is manifested as a spatially random texture and the lacunarity analysis of a spatially random binary process exhibits a characteristic rapid decay to the minimum value as window size increases; whereas, scene texture will exhibit a more complicated decay, if the sensor is indeed well-suited to reveal scene structure

Figure 1 illustrates the median Q1 lacunarity functions derived from 1000 samples at windows of 1 to 32 . The sharp change in slope in the May image corresponds to the emergence of a vegetation canopy after widespread flooding, which obscures the previously distinct spatial pattern of baias. In contrast to Q1, lacunarity functions for Q2 and Q3 (not shown) exhibit rapid decays to minimum values at all three dates indicating the spatial patterning of midrange values for backscattering are speckle-dominated. The Q4 pattern (not shown) as displays temporal variability but, unlike Q1, the May image exhibits a self-similar decay.

\section{Diversity}

The Shannon-Weaver-Wiener diversity index is a familiar information-theoretic measure widely applied in ecology, among other disciplines. Its application to imagery is straightforward once we consider each grey-level to be a distinct class. The diversity index describes the image in terms of the proportional representation of each grey-level in the histogram. The index assumes stationarity in the data but random resampling of the image stratified by window size is a robust procedure for handling scene patchiness. By observing the behavior of the second-order statistics of the index's sampling distribution (e.g. CV, range) as a function of window size, critical scales of scene texture can be identified. If the dominant spatial structure of the scene is patchy or H-resolution (sensu Strahler et al. 1986), then the CV of diversity (CVD) as a function of increasing window size might develop as follows. Initially, CVD will be lower when windows fall within patches but will increase as sampling extent increases until a peak is reached that corresponds to the scale of maximum entropy -- the point at which the histogram of the image subsamples exhibit the most even representation across diversity values. CVD will then drop as larger window sizes encompass more area and more patches but fewer "new" grey level arrangements. In constrast, a spatially random scene would generate an essentially flat diversity curve.

Figure 2 illustrates the CVD curves derived from 1000 subsamples at each window size. Note the bimodality in the December curve that shifts to a nearly unimodal curve in February and collapses in the May scene. This pattern indicates a profound shift in the spatial structure of greylevel values from a critical scale of about 80 ha to an essentially scaleless random structure.

\section{Multi-scale GLCM measures}

To attenuate the shortcomings of traditional singlescale GLCM measures, we randomly resampled the image $(n=1000)$ across a range of window sizes. Normalized GLCM measures were calculated (contrast, entropy, homogeneity, inverse difference moment, and uniformity) for horizontal (west-east), vertical, and the two diagonal first-order transitions in each window and sampling statistics were generated. GLCM measures were normalized to

enable comparisons across window sizes and images. If there is a characteristic scale at which grey-level transitions exhibit a strong pattem, then this GLCM "scoping" should be able to identify it. However, if most grey-level transitions are dominanted by noise, then no pattem across scales may emerge. Note that GLCM measures look for patterns in the matrix of grey-level transition probabilities rather than the image itself. The formulation of the matrix assumes spatial stationarity in the image. 
Figure 3 illustrates a typical result from the GLCM measures: little sensitivity to temporal changes in spatial structure. The entropy measure was selected to display since its formulation is closest in concept to the diversity measure. Little sensitivity to anisotropy was displayed in the GLCM measures and second-order statistics, such as $\mathrm{CV}$, also did not illuminate spatial structure

\section{DISCUSSION}

Of the three multi-scale approaches used, the multiscale extension of the traditional GLCM measures show little sensitivity to striking changes in the SAR images of the Pantanal landscape. Furthermore, GLCM measures are computationally intensive; whereas, better performance was obtained with a simple multi-scale sampling of an aspatial diversity measure. Lacunarity also exhibited good sensitivity to seasonality in backscattering but requires preprocessing to binary images. Lacunarity offers the power to distinguish between speckle and scene texture. In sum, both lacunarity and diversity offer new ways to quantify texture robustly in the face of spatio-temporal variability.

\section{REFERENCES}

Alho, C.J.R., Lacher Jr., T.E., Gonçalves, H.C.

"Environmental degradation in the Pantanal ecosystem." BioScience 38(1988):164-171.

Barber, D.G., and Ledrew, E.F. "SAR sea ice discrimination using texture statistics: a multivariate approach." Photogrammetric Engineering and Remote Sensing 57(1991):385-395.

Haralick, R.M., and Shapiro, L.G. Computer and Robot Vision, Volume 1. Reading, Massachusetts: AddisonWesley, 1992

Henebry, G.M. "Detecting change in grasslands using measures of spatial dependence with Landsat TM data." Remote Sensing of Environment 46(1993):223-234.

Plotnick, R.E., Gardner, R.H., and O'Neill, R.V. "Lacunarity indices as measures of landscape texture." Landscape Ecology 8(1993):201-211.

Rignot, E., and Kwok, R. "Characterization of spatial statistics of distributed targets in SAR data" Intemational Journal of Remote Sensing 14(1993):345-363.

Rizzini, C.T., Coimbra Filho, A.F., Houaiss, A. Ecossitemas Brasileiros/Brazilian Ecosystems. Rio de Janeiro: Index Editora, 1988.

Sheen, D.R., and Johnston, L.P. "Statistical and spatial properties of forest clutter measured with polarimetric synthetic aperture radar (SAR)." IEEE Transactions on Geoscience and Remote Sensing 30(1992):568-588.

Strahler, A.H., Woodcock, C.E., and Smith, J.A. "On the nature of models in remote sensing." Remote Sensing of Environment, 20(1986):121-139.

Ulaby, F.T., Kouyate, F., Briscoe, B., and Williams, T.H.L. "Textural information in SAR images" IEEE Transactions on Geoscience and Remote Sensing GE-24(1986):235245.
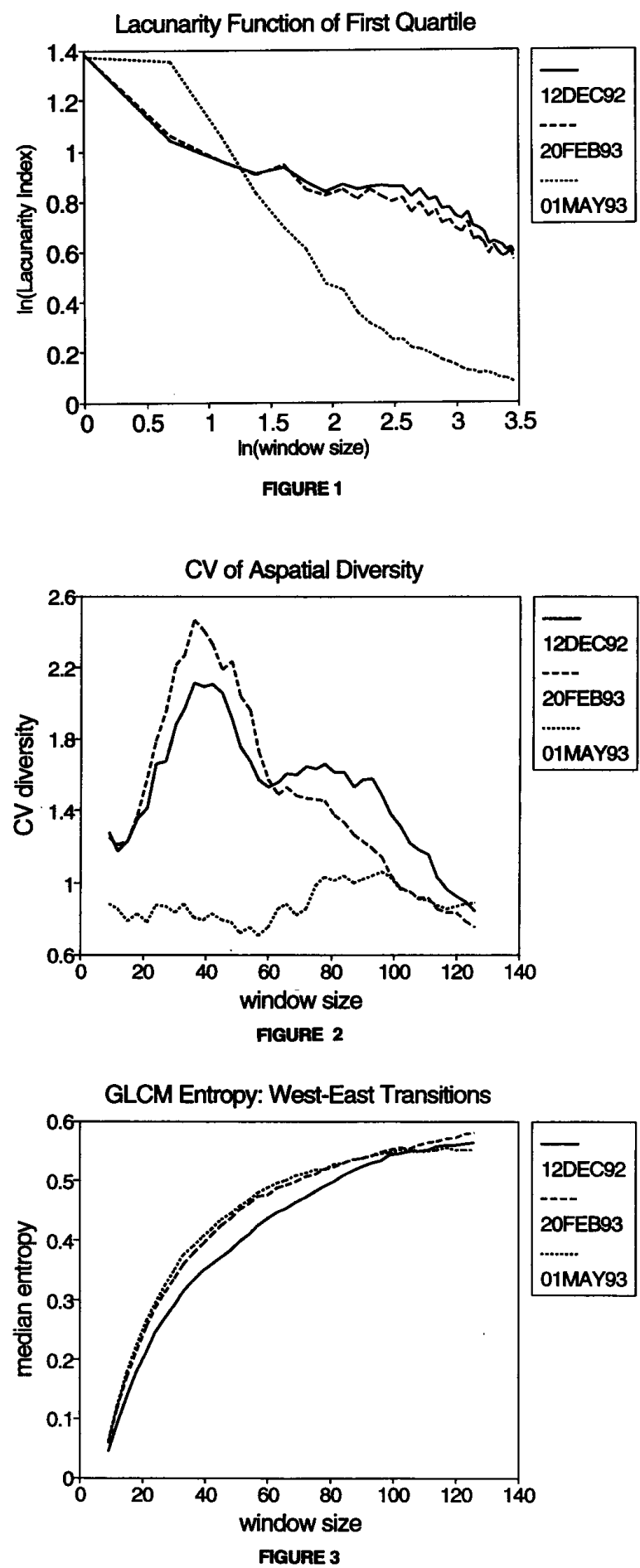